\title{
Criminologie
}

\section{Socialisation professionnelle des policiers : le rôle de l'école}

\section{Catherine Gorgeon}

Volume 29, numéro 2, automne 1996

L’homicide conjugal au Canada

URI : https://id.erudit.org/iderudit/017393ar

DOI : https://doi.org/10.7202/017393ar

Aller au sommaire du numéro

Éditeur(s)

Les Presses de l'Université de Montréal

ISSN

0316-0041 (imprimé)

1492-1367 (numérique)

Découvrir la revue

Citer cet article

Gorgeon, C. (1996). Socialisation professionnelle des policiers : le rôle de l'école. Criminologie, 29(2), 141-163. https://doi.org/10.7202/017393ar

\section{Résumé de l'article}

Anglo-Saxon studies on professional socialization of police officers have proven that the socialization process can be broken down into many steps, the major one being schooling. The results of all these studies have also

demonstrated that the existence of specific cultural characteristics in the police profession influence and model new recruits. Recent French studies, with more quantitative methods, confirmed some of these results, but show many nuances and insist on the diversity in attitudes and expectations on the part of police students. It is namely the case with the year of training for constables. 
Anglo-Saxon studies on professional socialization of police officers have proven that the socialization process can be broken down into many steps, the major one being schooling. The results of all these studies have also demonstrated that the existence of specific cultural characteristics in the police profession influence and model new recruits. Recent French studies, with more quantitative methods, confirmed some of these results, but show many nuances and insist on the diversity in attitudes and expectations on the part of police students. It is namely the case with the year of training for constables.

Depuis les travaux fondateurs de Westley (1953) et de Skolnik (196566), plusieurs recherches françaises portant sur la socialisation professionnelle des policiers ont fait avancer la problématique, et celle-ci est à présent suffisamment féconde pour pouvoir être discutée.

En bref, que nous disent les auteurs anglosaxons sur la socialisation professionnelle?

Grâce à Westley, Skolnik (op. cit.) et Neiderhoffer (1967) et peu après Sterling (1972), Harris (1973), Tifft (1974), Bennett (1975) et Van Maanen (1975), puis plus récemment Fielding (1988) en Grande-Bretagne, il est admis l'existence d'une "personnalité de travail» policière, d'un type unique d'attentes comportementales ou de normes professionnelles qui différencieraient les policiers du public. Deux hypothèses explicatives concurrentes sont généralement avancées :

- la première suggère que les individus autoritaires sont, d'une certaine manière, attirés par le travail de la police parce que ce rôle est compatible avec leurs forces, compétences, intérêts et besoins (Hanewicz);

- la deuxième soutient que les différences d'attitudes et de valeurs entre les policiers et les citoyens sont dues à l'exigence de l'emploi. Cette hypothèse de socialisation insiste à la fois sur la nature de l'emploi et sur le processus par lequel les recrues extraites de la population générale deviennent des policiers expérimentés avec une personnalité «policière».

1. Chargée de recherches, Institut des hautes études de la sécurité intérieure, 19 , rue Péclet, 75015 Paris, France. 
Skolnik, largement repris par nombre d'auteurs nous dit, quant à lui, deux choses à propos de la culture professionnelle policière :

- Tout d'abord, il insiste sur ce qui fait, selon lui, la spécificité de l'environnement policier: la combinaison de trois éléments - le danger, la nécessité de l'imposition de l'autorité et l'efficacité qui chacun considéré séparément ne sont pas une propriété spécifique du travail policier mais qui, combinés, produisent une personnalité de travail qu'il définit comme une prédisposition générale à percevoir et à agir d'une certaine façon.

- Deuxièmement, il relève neuf points constitutifs de cette «working personality » en insistant sur le fait qu'elle est commune et universelle quels que soient le corps et le grade auxquels appartient le policier. Ces neuf points sont: le soupçon, l'isolement social, la solidarité interne, un malentendu puissant entre la police et le public, l'exercice de l'autorité, un rapport ambigu à la morale, la fermeture sur le corps, la tendance au conservatisme, la pression informelle à l'efficacité.

Les exigences de l'organisation et la nature de l'emploi conduiraient ainsi à une unicité. D'autres affirment que ce serait au contraire l'environnement politique qui serait déterminant. En fait, ce qu'il faut retenir de ces travaux c'est qu'ils se fondent sur le postulat d'une relation linéaire entre une situation de travail et des valeurs professionnelles qui en seraient le produit.

Ces travaux ${ }^{2}$ font également clairement ressortir que le processus de socialisation se décompose en plusieurs étapes ( 3 ou 4 selon les auteurs) qui vont transformer la jeune recrue en «patrouilleur» (l'entrée, l'introduction, la rencontre, la métamorphose, Van Maanen, 1975). Ces phases vont impliquer des changements individuels manifestés, entre autres, à travers le rattachement au le groupe professionnel de référence et son influence. Enfin, les caractêristiques individuelles vont retarder ou accélérer le processus.

Le processus de sélection des futurs élèves gardiens n'est pas sans effet sur la construction des références professionnelles des jeunes gardiens de la paix. Van Maanen a observé que la sélection est un travail de socialisation important : entre l'examen et le coup de fil qui indique à la recrue qu'elle est acceptée dans le département ${ }^{3}$, l'individu passe à travers toute une série d'événements qui servent à cautionner l'idée qu'il est accepté dans une organisation d'élite. La procédure de sélection, que certains appellent socialisation anticipée, garantit que ceux qui rejoignent la profession

2. Ces enquêtes se sont déroulées chaque fois selon la même méthode : étude longitudinale par questionnaire et observation participante pendant la scolarité.

3. Aux États-Unis chaque «Police Department » municipal a la charge du recrutement et de la formation des policiers locaux. 
ont une attitude positive forte vis-à-vis de leur nouvel emploi (van Maanen, 1975). En effet, entre le moment où la potentielle future recrue va déposer son dossier et celui où l'agrément lui sera communiqué, il s'écoule au moins six mois pendant lesquels la démotivation peut naître, avec entre autres la perspective de rechercher d'autres possibilités d'embauche ou, à l'inverse, cette période peut laisser place à la recherche de contacts et d'informations pour se familiariser avec son futur métier. C'est pourquoi van Maanen met par ailleurs en évidence l'importance, pendant ce temps d'attente, des réseaux familiaux ou des proches appartenant déjà à l'institution et qui vont développer chez le policier potentiel un pré-attachement à l'institution avant même qu'il ne la rejoigne effectivement.

En ce qui concerne les motivations relatives à une carrière dans la police, van Maanen estime que les aspects de sécurité et de salaires de la profession ont sans doute été surestimés dans la littérature : il apparaît par contre chez les recrues qu'il a interrogées et observées une ambition élevée de servir la communauté et un attrait certain pour les qualités extérieures et présumées aventureuses du travail de police. Fielding note de son côté que les aspects du travail qui semblent aux recrues caractéristiques de l'emploi sont le lieu - la rue et non le poste - et les horaires de travail en dehors du contexte classique du travail de bureau ou en usine, et que la raison d'entrée la plus souvent citée par les recrues est la variété.

L'intérêt de faire un travail non routinier serait ainsi un élément important dans le choix du métier de policier. Van Maanen postule que la plupart n'ont pas choisi leur carrière au hasard, mais qu'ils entrent au départ avec un fort degré d'identification normative à ce qu'ils perçoivent comme les buts et les valeurs de l'organisation. C'est pourquoi les conditions, le contenu et la forme de l'enseignement sont primordiaux dans le processus de socialisation et d'apprentissage des valeurs policières.

La deuxième étape que constitue l'admission à l'école est en réalité le premier contact réel avec la sous-culture policière. C'est l'occasion pour les recrues de commencer à absorber la tradition qui caractérise le département de police. Van Maanen insiste sur l'importance de ce qu'il appelle les «récits de guerre», ainsi que sur le rôle de la soumission infligée par l'école, dont la discipline est particulièrement dure. Ce qui se passe à l'école est plus que la socialisation formelle. La vie sociale exclusivement tournée vers le groupe, le contact avec les instructeurs et les policiers expérimentés est une source de connaissance des principes essentiels de la culture policière (Fielding, 1988). Très vite, la recrue apprend l'importance que revêt son groupe de pairs et va s'identifier rapidement à ses camarades. La période de formation à l'académie sert aussi à préparer les recrues à changer leurs attentes professionnelles initialement élevées mais irréalistes. Ahern, lui, n'hésite pas à affirmer que l'activité policière est un travail qui 
demande un changement d'identité ${ }^{4}$ et que «le jour où la nouvelle recrue passe la porte de l'académie de police, elle quitte la société pour entrer dans (une profession qui fait plus que lui donner un travail, qui lui définit ce qu'elle est » (Ahern, 1972:3).

C'est donc lors de son premier contact avec l'institution policière (i.e. à l'école) que la recrue découvre les valeurs professionnelles, qui peuvent du reste être remises en cause lors de son «entrée» sur le terrain où se fait tout un travail de régulation. Harris, van Maanen et Niederhoffer suggèrent que l'expérience de terrain, après l'académie, produit un réel état de choc. Les attentes générées à l'école sont généralement radicalement modifiées une fois que la recrue commence le travail de terrain. Le jeune policier apprend très vite qu'en réalité, la plus grande partie de son travail sera routinier et qu'il est avant tout un membre réactif d'une organisation de service. Puis, l'expérience s'accumulant, la répétition d'événements routiniers et la longue attente pour l'inhabituel vont caractériser l'expérience de socialisation postérieure (Fielding, 1988).

Bien sûr, les caractéristiques personnelles des individus vont accélérer ou retarder le processus de socialisation professionnelle dont on peut dégager, grossièrement trois composantes complémentaires: la nature de la "socialisation anticipée» (i.e. tout ce qui s'est passé avant l'entrée à l'école); la nature des rencontres policières; les facteurs structurels (poids de la hiérarchie, importance des tâches de "paperasse ", nombre de règles et règlement...)

Skolnick a mis en évidence le fait que l'engagement professionnel fort des recrues implique une croyance aveugle dans le système organisationnel et son acceptation totale. Par engagement professionnel, il entend la loyauté envers l'institution, la bonne volonté pour recommander l'institution comme un bon endroit pour travailler, la bonne volonté pour aider l'institution à réussir... Or, cet engagement décroît très rapidement et de façon significative même si, malgré cette tendance à la baisse, van Maanen note dans l'absolu que le niveau d'engagement reste relativement élevé par rapport à d'autres professions (van Maanen, 1975).

Une des conclusions des travaux de cet auteur est que « Progress along the socialization continuum in the police world can be seen as a gradual development of an "in the same boat" consciousness stressing a "don't make wave" occupational philosophy " (van Maanen, 1975). Le jeune patrouilleur découvre que la solution la plus satisfaisante par rapport au labyrinthe de la hiérarchie, la paperasserie et la frappe, la pléthore de règles et de régulations et le sale boulot qui caractérise l'emploi est d'adopter la norme du groupe.

4. Voir également Fielding. 
Cela suggère que les déceptions des policiers sont de deux ordres : vis-à-vis du public et du système. Les recrues réalisent que ce sont les relations avec les collègues qui protègent leurs intérêts et leur permettent de continuer le «boulot».

En conséquence, selon ces auteurs, «the police culture can be viewed as molding the attitudes - with numbing regularity - of virtually all who enter» (van Maanen, 1975). Autrement dit, au fur et à mesure qu'elles acquièrent une connaissance du travail, les recrues vont, sous l'influence des caractéristiques culturelles de la profession, modifier la façon dont elles considèrent l'organisation (Fielding, 1988). Certes, Fielding notamment, parle à quelques reprises de plusieurs «sous-cultures » professionnelles, de négociation, entre l'influence de la culture professionnelle et les propres perceptions individuelles, et indique qu'«il ne faut pas oublier qu'entrer dans la police n'est pas automatiquement entrer dans la culture, que certains policiers ne veulent pas et ne voudront jamais adhérer à cette culture, qu'il y a plus d'une culture policière et que les décisions individuelles sont toujours l'expression d'une perspective individuelle. " Mais aucune hypothèse n'est avancée sur les éléments structurant ces différentes souscultures.

Bien qu'il soit difficile de faire totalement abstraction des différences existant dans les processus de formation des policiers français et anglais ou américains, les recherches françaises sur la socialisation professionnelle, en même temps qu'elles confirment un certain nombre de résultats, mettent en évidencè un constat beaucoup plus nuancé.

Je ferai référence à trois principaux travaux qui sont, par ordre chronologique :

- une enquête réalisée en 1982 par la société Interface auprès de 70000 des 110000 policiers de l'époque, questionnaire qui portait essentiellement sur la formation (Hauser, Tiévant, 1985);

- une enquête réalisée entre octobre 1983 et septembre 1984 auprès de 96 élèves gardiens de la paix (quatre promotions de la même école) interrogés une première fois à leur entrée au centre de formation et une seconde fois (même questionnaire) à l'issue de la scolarité, six mois après (Loubet del Bayle, 1988);

- la troisième recherche, en cours, est l'étude longitudinale d'une promotion entière d'élèves gardiens de la paix (soit environ 1000 individus) réinterrogés à trois reprises pendant leur scolarité - qui est actuellement de 12 mois -, puis périodiquement une fois par an après leur affectation dans leur service; la procédure de raccordement des questionnaires permet, au-delà des différences nettes, de saisir des variations croisées, actuellement, seuls les résultats des 
trois premières interrogations réalisées entre le début et la fin de la scolarité ont été exploités (Monjardet, Gorgeon, 1992, 1993).

\section{DES ÉVOLUTIONS GLOBALES SANS SURPRISE...}

Les résultats d'ensemble de ces recherches metteni en évidence, entre le début et la fin de la scolarité, des évolutions globales proches de celles constatées par les chercheurs anglo-saxons.

Premièrement, un certain désenchantement et un remodelage des attentes par rapport au métier, avec :

— une très forte baisse du sentiment d'utilité, une augmentation de la crainte d'inefficacité ;

- une forte croissance des motivations utilitaires;

- une croissance du sentiment de spécificité du métier;

- une tendance à assumer plus difficilement certains aspects de la fonction (port de l'uniforme, manière d'annoncer son métier ${ }^{5}$ );

- une baisse du dynamisme des élèves (refus, par exemple, de prendre des initiatives afin de ne pas avoir d'ennuis).

Deuxièmement, à l'égard du public, on note une tendance à l'accroissement de la méfiance à l'égard de l'environnement sociétal (Loubet del Bayle, 1988).

Monjardet et Gorgeon notent, certes, l'adoption massive d'un petit nombre de stéréotypes mais le trait le plus net, à l'encontre de ce qu'affirme la littérature anglo-saxonne, est sa faible extension: le nombre des questions qui fait l'unanimité est faible.

Ces stéréotypes se retrouvent dans trois domaines essentiels :

- la première qualité requise du policier, l'honnêteté, est celle sur laquelle les recrues se rallient massivement à la sortie de l'école (35\% des répondants à l'entrée, à l'école, contre $61 \%$ à la sortie; et l'honnêteté gagne cinq fois plus de suffrages;

- l'image de la police et le rapport aux médias : les recrues sont $94 \%$ à la sortie de l'école contre $74 \%$ à l'entrée à penser que les médias donnent une image défavorable de la police :

- enfin, un troisième domaine relatif aux propriétés du métier luimême : l'importance de la solidarité s'accroit ${ }^{6}$ et prend le pas sur

S. La question exacte était : $\mathbf{S i}$, dans une conversation, on vous demande quel est votre futur métier, vous répondrez : 1) fonctionnaire, 2) fonctionnaire de police, 3) gardien de la paix, 4) policier, 5) vous ne répondrez pas précisément.

6. Celle-ci était déjà plus élevée chez les policiers auxiliaires, indiquant par là que leur passage dans la Police nationale a eu pour conséquence l'intériorisation de certains stéréotypes de la profession. Pour plus de développement sur ce sujet voir Monjardet, D., note sur les P.A. en formation, IHESI, février 1994. 
l'ambiance de travail et le respect du règlement ${ }^{7}$; de même qu'aug mente l'idée d'une contradiction possible entre la recherche d'efficacité et le respect du règlement.

Dans ces domaines, comme l'a justement observé van Maanen, il est clair que l'enseignement formel y est pour peu de chose, et c'est bien du côté de l'environnement du policier en formation qu'il faut aller chercher des éléments explicatifs (au sein même des recrues avec les policiers auxiliaires et à l'intérieur de l'école à travers les discours des formateurs, du directeur, les postes de gardes, les unités d'actifs présentes sur le site ${ }^{8} \ldots$ )

En fait, trois mouvements dominent quand on observe les variations majeures pour l'ensemble de la promotion étudiée entre l'entrée à l'école de police et la sortie, et qui se rapprochent également des résultats anglosaxons :

1) le premier est la conséquence directe d'une meilleure information sur les réalités du métier, on note un réaménagement des attentes et des projets initiaux, marqué par un plus grand réalisme.

2) ce réalisme accru s'accompagne d'un désenchantement, qui porte plus sur l'institution policière (l'administration Police nationale) que sur le métier lui-même.

3) la troisième évolution importante porte sur la dimension relationnelle du métier et la relation entre la police et le public : on avait noté à l'entrée à l'école une très grande ouverture des recrues, qui les différenciait très nettement des policiers en activité; à la fin de la scolarité, les élèves définissent les missions de police de façon plus restrictive, valorisent moins les qualités de contact et font preuve d'une plus grande défiance à l'égard du non-policier.

Les policiers auxiliaires sont des garçons qui ont effectué leur service national dans la police : ils ont eu deux mois de «classes", c'est-à-dire de formation, et ont ensuite passé huit mois en service actif. Certains d'entre eux, après cette expérience, passent le concours de gardiens de la paix. Ils suivent la même scolarité que les autres recrues.

7. La question précise était : Selon vous, le plus important dans le métier de gardien de la paix, c'est surtout...

1- de faire un travail intéressant;

2- de travailler dans une bonne ambiance ;

3- de pouvoir compter sur les collègues ;

4- de respecter le règlement.

8. Malheureusement, pour des raisons techniques, la recherche dont il est question s'est privée du matériau précieux qu'auraient constitué l'observation du dispositif de formation initiale et des entretiens, moins directifs que le seul questionnaire, tant auprès des élèves que de leurs formateurs. 


\section{... QUI MASQUENT DES ATTITUDES ET DES ATTENTES BEAUCOUP PLUS DIVERSIFIÉES}

Mais l'intérêt de la méthode, grâce aux données dont nous disposons ${ }^{9}$, est de permettre de travailler non seulement sur des soldes qui peuvent masquer des mouvements inverses qui se compensent mais sur des variations croisées ${ }^{10}$. Et ce qu'indiquent les données est alors beaucoup plus nuancé.

Certes, en fin de scolarité, d'une part, les propriétés des recrues s'estompent pour laisser la place à une très nette homogénéisation des opinions dans certains champs. Grâce à la formation, elles ont une idée plus précise de ce qui les attend concrètement et, d'autre part, l'image du métier chez les élèves a évolué positivement. Néanmoins, en fonction de l'affermissement ou, au contraire, de l'effritement de la vocation, les modalités d'investissement professionnel varient: ainsi, les recrues qui se font du gardien de la paix l'image du «spécialiste de la voie publique» sont $67 \%$ à récuser le conseil emblématique de van Maanen «ne fais pas de vague c'est la meilleure façon de ne pas avoir d'ennuis», tandis qu'elles sont $58 \%$ à l'approuver parmi celles qui voient le gardien de la paix comme l' " homme à tout faire de la Police nationale »; du reste, ces mêmes élèves appréhendent de plus en plus le métier en terme d'emploi et de conditions de travail.

L'enquête d'Interface est en fait la première chronologiquement à avoir dégagé les grands axes de construction d'une typologie que les recherches suivantes ne feront qu'affiner. Elle met au jour un premier axe intitulé «ouverture-repli» qui, à un pôle, distingue les policiers exprimant une certaine méfiance à l'égard des autres en général et à l'autre pôle, au contraire, ceux qui expriment une envie de communiquer davantage avec l'extérieur.

9. Elles consistent en 951 ensembles de trois questionnaires successifs (à l'entrée à l'école pendant la première semaine, neuf mois après et à la sortie de l'école) attribuables au même répondant. Pour une présentation détaillée de l'enquête, voir Monjardet, D., Gorgeon, C., La socialisation professionnelle des policiers -Étude longitudinale de la $12 \mathrm{~J}^{e}$ promotion des élèves gardiens de la paix. Tome 1 , La formarion initiale, Paris, Institut des Hautes Études de la Sécurité Intérieure, 1993.

10. Par exemple, à la question «Si vous aviez eu le choix, auriez-vous choisi un autre emploi? » apparemment $13 \%$ des recrues ont changé d'avis entre l'entrée et la sortie de l'école : elles sont $30 \%$ en janvier puis $43 \%$ en décembre à indiquer que si elles avaient pu elles auraient opté pour une autre profession. En réalité, c'est plus d'un quart des élèves qui ont inversé leurs réponses : $7 \%$ ont été en quelque sorte "convaincus » par la scolarité et en décembre affirment que s'ils avaient eu le choix, ils seraient tout de même entrés dans la police (ils disaient l'inverse en janvier), et $20 \%$ ont fait le cheminement inverse et peuvent être considérés comme « déçus » par leur choix professionnel. 
Un deuxième s'articule autour de «satisfaction-insatisfaction» : celuici n'est pas spécifiquement policier : il se retrouve dans bien d'autres secteurs professionnels. Néanmoins, il semble être lié au positionnement des policiers sur les deux autres axes étant ainsi, en quelque sorte, moteur pour les deux autres.

Enfin, un troisième axe dénommé «réglementarisme-négociation » oppose les policiers privilégiant la concertation et ceux préférant les actions de contrôle et d'autorité ${ }^{11}$. Ce que l'étude Interface met en évidence, c'est donc bien «les dimensions communes sur lesquelles les policiers se partagent. " (Monjardet, 1994 ${ }^{12}$ ) et non des traits communs.

De même, ce qui est intéressant dans l'enquête de Loubet del Bayle, ce n'est pas tant de voir comment, à la sortie, les élèves ont modifié leurs opinions à la fois sur leur métier et sur leurs rapports avec le public, mais d'avoir mis en lumière l'importance d'un certain nombre de variables structurant différentes attitudes par rapport au métier et à la relation avec le public. Loubet del Bayle, à partir du calcul de plusieurs indices : de «vocation »; «relationnel»; «distance»; de «responsabilité »; « repliement » dégage une typologie à quatre pôles :

- les «responsables»: préoccupés par leurs rapports futurs avec le public et conscients de la dimension relationnelle du métier;

— les «repliés" conscients de l'utilité sociale de la police, mais avec une forte tendance au repliement (pensent que le public les gênera ou les désapprouvera, que le fait d'appartenir à la police rendra leurs voisins distants ou qu'ils ne connaîtront pas leur métier, qu'il est normal que la police garde le secret sur les motifs d'un contrôle d'identité, que les causes des critiques de la police par le public sont dues pour l'essentiel à l'influence des médias ou aux préjugés mêmes du public, etc.);

— les «relationnels»: montrent un fort degré d'engagement dans le métier, un intérêt pour les contacts humains, le sens des problèmes relationnels et une ouverture sur l'extérieur;

— les «sereins»: ne s'inquiètent ni de la dimension relationnelle ni des problèmes concernant les rapports police/public.

Loubet del Bayle indique aussì «une tendance à ce que les évolutions constatées différencient selon les attitudes individuelles des élèves à l'entrée dans le processus de formation et selon les tendances globales des promotions auxquelles ils appartiennent.»

11. Pour un développement plus long, voir Monjardet, D., Intervention au séminaire sur la déontologie, lHESI, 1992.

12. Monjardet, D., La culture professionnelle des policiers, in Revue Française de Sociologie, XXXV, 1994, pp. 393-411. 
Ainsi, est déjà avancée l'hypothèse selon laquelle on ne peut considérer les futurs policiers comme un groupe homogène mu par les mêmes «dispositions» vis-à-vis du métier et doté de la même appréhension de la société. De même, l'apprentissage, et en particulier le passage à l'école ou à l'académie, est loin de laisser sur tous les mêmes traces qui permettraient de conclure à un processus universel de socialisation professionnelle (Monjardet, Gorgeon, 1993).

Notre troisième enquête est à ce sujet la plus probante. Outre qu'elle remet en cause fondamentalement l'existence d' «une» culture policière unique et homogène, elle démontre que la période de formation, première étape dans le processus de socialisation professionnelle, sera reçue diversement par les recrues et que celles-ci se partagent selon l'expérience sociale, les motivations de départ, les représentations du métier et les attentes professionnelles, mais aussi selon ces deux dimensions essentielles du métier que sont le rapport à l'autre et à la loi (Monjardet, Gorgeon, 1993).

L'exploitation du premier questionnaire, soumis aux élèves à leur entrée à l'école (Monjardet, Gorgeon, 1992), si elle met en évidence trois points communs qui rapprochent les élèves - la proximité ${ }^{13}$ à l'égard du monde policier, un très grand optimisme professionnel ${ }^{14}$ et un accent majeur mis sur les dimensions relationnelles du métier ${ }^{15}$-, indique surtout, à l'encontre de ce qu'affirment les auteurs américains, que la promotion témoigne du pluralisme des opinions, attentes et représentations que l'on peut attendre de la part d'un échantillon de jeunes qui ne se caractérisent pas tous par une vocation précoce et affirmée du métier de policier. L'enquête met en évidence une série de critères qui différencient dès le départ les recrues: le sexe, les motivations du choix professionnel, l'expérience policière et les représentations de la loi et de l'ordre. Ce demier critère, nous allons le voir, est essentiel : de façon très nette, il apparaît une différenciation au sein des recrues entre trois représentations de la loi et plus généralement de la norme. Pour certaines, c'est avant tout une contrainte, si

13. Plus du quatre cinquièmes des recrues connaissaient personnellement un policier ou un gendarme avant de tenter le concours et plus du tiers par lien de parenté.

14. Les recrues témoignent de très fortes attentes et d'une grande confiance dans l'administration dans laquelle elles entrent. Par exemple, à l'égard de leur avenir dans l'administration et des possibilités de carrière et de promotion, elles font preuve d'une remarquable unanimité : $95 \%$ pensent que la Police nationale leur permettra de réaliser leurs souhaits de carrière professionnelle et $99 \%$ se déclarent prêts à s'engager dans les concours intemes.

15. Qu'ils conçoivent comme un métier de généraliste, polyvalent. Dans le même principe, on note une forte adhésion au principe d'enseignements communs avec d'autres partenaires de la sécurité et à celui de stages pratiques dans d'autres services publics. À l'inverse, le modèle d'une police beaucoup plus resserrée sur des missions de répression de la délinquance et plus distante du public est très minoritaire. 
ce n'est un obstacle à l'efficacité policière, pour d'autres c'est un cadre nécessaire ou inévitable, pour les derniers, enfin, c'est la transcription ou la codification d'un ensemble de valeurs. Ces différentes acceptions de la loi oriehtent fortement certaines représentations du métier et des missions de police.

Et, à l'issue de la scolarité, ce qu'indiquent les données, c'est d'une part le pluralisme maintenu des recrues sur l'ensemble des variables significatives dans la définition de la police, de ses missions, des métiers qui y correspondent, des rapports qu'elle doit entretenir avec l'autre et avec la loi et d'autre part le caractère structurant de ces deux dernières dimensions du rapport à la loi et du rapport à l'autre en ce sens que «... [ce] sont les deux enjeux majeurs sur lesquelles les recrues se positionnent, et de leur position dans ces champs découlent leurs positions dans les autres domaines de l'activité professionnelle, aussi bien que la forme et, l'intensité de l'investissement qu'ils opèrent dans leur métier» (Monjardet, 1994). La formation reçue ne sera donc pas appréciée et enregistrée de la même manière par l'ensemble des élèves: certains y auront trouvé ce qu'ils attendaient, d'autres se trouvent finalement plutôt bien préparés à leur métier alors même qu'ils ne cachaient pas leur scepticisme à l'entrée, d'autres encore ont, à l'inverse, été déçus et enfin, quelques-uns sont restés à l'entrée et à la sortie négatifs quant à cette année de formation initiale.

\section{L'APPRÉCIATION DE LA FORMATION...}

\section{Les attentes initiales en matière de formation}

Celles-ci étaient très fortes et très positives puisque, à l'entrée à l'école, plus de 7 recrues sur 10 (71\%) pensent que grâce à la formation elles vont «être bien préparées à affronter les différents contextes dans lesquels [elles] devront intervenir et à comprendre les différents publics auxquels [elles] auront affaire». Or, en fin de scolarité, cette formation ne rallie plus que $51 \%$ des suffrages ${ }^{16}$. Visiblement, certaines attentes ont été déçues, qu'il s'agisse des contenus, des méthodes ou des formateurs.

En ce qui concerne les attentes en matière de formation, nous avions distingué entre autres : 1) des connaissances juridiques et administratives, 2) des rencontres avec des policiers, 3) des techniques policières, et enfin

16. Les élèves ne sont plus que $51 \%$ contre $71 \%$ à penser que leur formation les a bien préparés à affronter les différents contextes dans lesquels ils devront intervenir et à comprendre les publics auxquels ils auront affaire. 
4) des renseignements sur l'organisation et le fonctionnement de l'institution ${ }^{17}$.

Globalement, sur l'ensemble de la promotion, la formation semble avoir été satisfaisante en matière de connaissances administratives et juridiques (parmi les recrues qui attendaient ces connaissances, le pourcentage de satisfaits est de $75 \%$ ) et les renseignements sur l'organisation et le fonctionnement de l'institution ont été suffisants (61\% de satisfaits parmi ceux qui attendaient avant tout de leur formation ce type de renseignements). Par contre, visiblement, les rencontres avec des policiers de terrain ont manqué (les recrues qui attendaient avant tout ces rencontres ne sont que $25 \%$ à être satisfaites de leur formation), et l'apprentissage des techniques policières laisse la moitié de la promotion insatisfaite.

Mais l'enquête montre aussi que, pour l'ensemble de la promotion, il y a eu, en fin de scolarité, un double mouvement : un déplacement des attentes d'une part, et un jugement plus négatif sur la formation reçue d'autre part, et ces deux mouvements sont liés. En effet, si à l'entrée à l'école, on ne relève pas de relation entre attentes en matière de formation et jugement (anticipé) sur la formation que les élèves pensent recevoir, à la fin de la scolarité, par contre, le jugement porté sur la formation ${ }^{18}$ est nettement corrélé avec la nature des attentes: les recrues qui attendaient avant tout de leur formation des connaissances administratives et juridiques ou des renseignements sur l'organisation et le fonctionnement de l'institution policière sont $56 \%$ à porter un jugement positif à l'égard de la formation reçue, tandis que les recrues qui attendaient avant tout des rencontres avec des policiers ou des techniques policières ne sont que $46 \%$ à avoir ce jugement favorable. En même temps, les attentes se sont déplacées à la fois vers les rencontres avec des policiers de terrain et vers les connaissances administratives et juridiques. Mais ces deux mouvements n'ont en réalité pas la même signification. En effet, ces attentes différenciées ont une influence très nette à la fois sur le taux de satisfaction et sur le jugement vis-à-vis de la formation. Ainsi, parmi les élèves qui affirment avoir attendu avant lout des rencontres avec des policiers de terrain, on trouve $76 \%$ d'insatisfaits, alors que chez les recrues qui attendaient surtout des connaissances juridiques et administratives, on compte à l'inverse $75 \%$ de

17. La question exacte était: Qu'attendiezvous d'abord de votre formation? 1) des connaissances administratives et juridiques, 2) des rencontres avec des policiers de terrain ou des récits sur le vrai métier de policier, 3) des techniques policières, 4) des renseignements sur l'organisation et le fonctionnement de l'institution policière, 5) des rencontres avec des personnes représentant l'environnement quotidien du policier, 6) des renseignements sur la façon de se comporter avec les publics.

18. À travers la question: Pensezvous que votre formation vous a bien préparé à affronter les divers contextes dans lesquels vous devrez intervenir et à comprendre les publics auxquels vous aurez affaire? 
satisfaits. Et, dans le même sens, les recrues en attente de rencontres sont moins nombreuses en fin de scolarité à trouver que leur formation les préparera bien à leur métier $(44 \%)$ que celles en attente de connaissances juridiques et administratives $(56 \%)$.

Il y a donc bien eu en fin de scolarité un réaménagement des attentes, mais pour les uns il exprime une frustration, pour les autres, au contraire, une adhésion au contenu de l'enseignement qu'ils ont reçu.

Les attentes envers les formateurs suivent le même modèle : au fur et à mesure de la scolarité, les recrues sont plus nombreuses à attendre de leur formateur une solide expérience professionnelle (la proportion passe de $45 \%$ à $55 \%$ ) aux dépens essentiellement des connaissances (le taux passe de $27 \%$ à $21 \%$ ).

De la même façon, c'est parmi les recrues qui attendaient avant tout de leurs formateurs des connaissances techniques et juridiques que l'on trouve le plus de satisfaits vis-à-vis de ces formateurs (73\%); parmi les recrues qui en attendaient une expérience professionnelle, le taux baisse à $59 \%$. Et, comme on pouvait s'y attendre, ces attentes en direction des formateurs jouent à la fois sur la satisfaction vis-à-vis de la formation et sur le jugement porté sur l'efficacité de la formation:

- les recrues qui attendaient d'abord de leurs formateurs des connaissances sont satisfaites de la formation à $64 \%$, et parmi elles $63 \%$ trouvent que la formation les a bien préparées;

- les recrues qui attendaient une expérience professionnelle ne sont plus que $44 \%$ à être satisfaites de la formation, et $47 \%$ seulement ont un avis positif sur la formation ${ }^{19}$.

\section{La préparation aux différentes tâches}

Une majorité relative de recrues $(41 \%)$ pense, en fin de scolarité, que c'est aux tâches de surveillance, contrôle, patrouille et verbalisation qu'elles sont le mieux préparées et, inversement, la tâche pour laquelle les recrues se sentent le moins préparées est le maintien de l'ordre ${ }^{20}$. Et lorsque l'on compare ces réponses avec celles formulées à l'entrée à l'école, on observe un déplacement dans le sens d'un plus grand réalisme, réalisme

19. Comme on pouvait s'y attendre, les policiers auxiliaires sont mieux informés des réalités du métier qui les attend. Leurs attentes en matière de formation en début de scolarité sont mieux ciblées et la majorité $(59 \%)$ attend d'abord des compétences (connaissances administratives et juridiques) et des techniques policières.

20. Les recrues ont compris que ce n'était pas en formation initiale à l'école qu'elles allaient apprendre le maintien de 1 'ordre. 
dont faisaient déjà preuve, dès le début de la scolarité, les policiers et les gendarmes auxiliaires ${ }^{2 l}$.

Autre remarque : de la même façon qu'à propos des attentes en matière de formation le déplacement des réponses vers des rencontres ou vers des connaissances administratives et juridiques n'a pas la même signification, ici le déplacement important des réponses vers la tâche libellée «remplir des papiers, écrire des rapports » est en réalité une appréciation négative. En effet, parmi les recrues qui, en fin de scolarité, pensent que c'est à cette tâche qu'elles seront le mieux préparées, $65 \%$ ne sont pas satisfaites de leur formation et $73 \%$ pensent que la formation ne les a pas bien préparées à ce qu'elles vont rencontrer (ce sont à chaque fois les taux les plus forts).

Quant aux critiques générales qui sont formulées à l'égard de la formation, elles sont de deux ordres. Soit sont mises en cause les limites mêmes de la formation et l'idée qu'on ne peut tout aborder quelle que soit l'intensité et la durée de la scolarité, compte tenu de l'infinie variété des tâches, des circonstances et des publics qui attendent les gardiens de la paix. Soit est mise en avant l'idée que l'apprentissage d'un tel métier ne peut se faire que sur le terrain et c'est alors le manque d'expérience professionnelle qui est invoqué. De ce point de vue, en fin de scolarité, le doute sur l'efficacité de l'enseignement formel a progressé dans l'ensemble de la promotion et est présent avec la même fréquence chez toutes les recrues alors qu'à l'entrée à l'école, il était surtout le fait des recrues ayant quelque expérience policière (policiers auxiliaires ou enfants de policier). Néanmoins, le clivage demeure toujours à la sortie de l'école entre les «expérimentés» (enfants de policiers et policiers auxiliaires) et les autres. Sans doute faut-il voir là un effet de la culture policière dont un des effets est de transmettre que ce n'est pas à l'école qu'on vous apprend le métier, mais que celui-ci s'acquiert sur le terrain.

On voit d'ores et déjà que la formation n'est pas perçue de manière identique par l'ensemble des recrues.

\section{... RÉVÈLE DES OPPOSITIONS PLUS PROFONDES}

À partir des deux questions relatives à la satisfaction des élèves vis-à$v$ is de la formation et de leurs formateurs en fin de scolarité, et de celle qui leur demande d'indiquer s'ils pensent que la formation les a bien préparés à leur métier ${ }^{22}$, on peut construire un indice de "contentement» décroissant

21. Comme les policiers auxiliaires, les gendarmes auxiliaires ont effectué leur service national dans la gendarmerie nationale.

22. La question précise était la suivante : Pensez-vous que votre formation va bien vous préparer (vous a bien préparé) à affronter les divers contextes dans lesquels vous devrez intervenir et à comprendre les publics auxquels vous aurez affaire? (réponse par oui ou non). 
et scinder la promotion en six groupes caractérisés selon le nombre de réponses positives à ces trois questions. À un bout de l'échelle, le groupe 1 $=$ les contents de tout $(+++=258$ ou $27 \%)$ à l'autre bout $6=$ les mécontents de tout $(--=182$ ou $19 \%)$.

Ces deux groupes les plus contrastés ( 1 et 6) s'opposent sur l'ensemble des questions concernant la formation mais également sur l'image du métier, l'investissement professionnel, la conception des missions de la police, le rapport à la loi. Les quelques chiffres qui suivent sont à eux seuls assez parlants.

Les «contents" sont entrés dans la police avec une vocation plus affirmée que les mécontents $(66 \%$ contre $58 \%)$ ) et surtout, celle-ci s'est maintenue jusqu'à la fin de la scolarité. Leurs attentes en matière de formation portaient surtout sur des connaissances administratives et juridiques ( $22 \%$ en janvier et $51 \%$ en décembre). De même, ils attendaient certes de leurs formateurs une solide expérience professionnelle - ce en quoi ils semblent plutôt satisfaits ( $43 \%$ en janvier, $47 \%$ en décembre) -, mais aussi des connaissances techniques et juridiques ( $30 \%$ en janvier et $31 \%$ en décembre). Dans des proportions importantes, ils sont moins nombreux que les mécontents à avoir trouvé la formation trop longue, à penser que la discipline est une contrainte inutile, que la pédagogie par objectifs morcelle la formation. Bref, ils ont adhéré au dispositif de formation et, du reste, se sentent plutôt bien préparés à surveiller, patrouiller, verbaliser, contrôler. Ce qui n'est pas le cas du groupe opposé, qui affiche plus souvent, en fin de scolarité, des mobiles utilitaires, qui attendaient avant tout de cette formation des rencontres avec des policiers et de leurs formateurs une solide expérience professionnelle et qui, visiblement, n'ont pas eu ce qu'ils espéraient : ils sont $23 \%$ (contre $7 \%$ chez les contents) à estimer qu'ils ne sont pas bien préparés à surveiller, patrouiller, contrôler, verbaliser et, par contre, ils s'estiment «trop bien » préparés en quelque sorte à des tâches qui leur paraissent secondaires telles que remplir des papiers $(26 \%$ contre $8 \%$ chez les contents).

De même, chez les contents, les messages véhiculés par la formation ont été entendus. Pour preuve, les résultats à la question relative à l'usage de l'arme. Ce qui n'est pas le cas chez les mécontents. 
Tableau 1:

Quelques chiffres relatifs à la formation en fonction de l'indice de contentement

\section{Groupe 1 Groupe 6}

Entrés dans la police pour des mobiles utilitaires

- janvier

- décembre

$33 \quad 42$

$34 \quad 48$

Attentes en matière de formation :

1. connaissances administratives et juridiques

$\begin{array}{lll}\text { - janvier } & 22 & 20 \\ \text { - décembre } & 51 & 42\end{array}$

Attentes en matière de formation en décembre :

2. rencontres avec des policiers

\begin{tabular}{lll} 
- janvier & 15 & 16 \\
- décembre & 15 & 21 \\
\hline
\end{tabular}

Attentes formateurs :

- solide expérience professionnelle

- janvier

$43 \quad 50$

- décembre

48

Attentes formateurs:

- connaissances techniques et juridiques

- janvier

- décembre

$30 \quad 23$

Formation trop longue

Discipline $=$ contrainte inutile

31

Discipline $=$ apprentissage nécessaire

$66 \quad 89$

$4 \quad 19$

La pédagogie par objectifs morcèle la formation

$67 \quad 47$

Se sentent le mieux préparés à :

- surveiller, patrouiller, verbaliser, contrôler

Se sentent le moins préparés à :

31

64

- surveiller, patrouiller, verbaliser, contrôler

$48 \quad 28$

$8 \quad 26$
7

Il est légitime d'utiliser son arme contre un véhicule en fuite* :
- janvier
- décembre
10

* Le mémento du gardien de la paix, qui a valeur réglementaire pour la profession précise bien que "pour arrêter un véhicule, seuls les moyens matérieis réglementaires peuvent être utilisés (panneaux luminescents "halte police", signaux optiques et herses le cas échéant) » et que "le seul fait pour un automobiliste de ne pas obéir à ces injonctions n'autorise pas l'usage de l'arme (contrairement au règlement de la Gendarmerie nationale dans ce domaine). L'emploi de l'arme dans ces circonstances reste soumis aux conditions générales de la légitime défense ", définies par ailleurs (il faut une agression injustifiée, une attaque caractérisée et non la simple crainte d'une éventuelle violence, il faut que la défense soit proportionnée à l'attaque. 
La formation a permis aux recrues de mieux cerner l'image du gardien de la paix: pour les contents, il est avant tout un spécialiste de la voie publique (à peine encore $4 \%$ d'entre eux le voient encore en fin de scolarité comme un simple exécutant). Du reste, leur image du métier a évolué positivement pour une majorité d'entre eux $(52 \%)$ et leur image de la Police nationale s'est nettement moins dégradée que chez le groupe opposé. Et naturellement, c'est le groupe des mécontents qui trouve le plus à redire de la formation des policiers en général, de leur motivation et de l'organisation des services.

Tableau 2 :

Quelques chiffres relatifs à l'image du gardien de la paix et du métier

\begin{tabular}{|c|c|c|}
\hline & Groupe 1 & Groupe 6 \\
\hline \multicolumn{3}{|l|}{ L'image du gardien de la paix : } \\
\hline \multicolumn{3}{|l|}{ - spécialiste de la voie publique } \\
\hline- janvier & 49 & 41 \\
\hline \multirow{2}{*}{ - décembre } & 59 & 35 \\
\hline & +10 & -6 \\
\hline - simple exécutant & 10 & 15 \\
\hline - janvier & 4 & 15 \\
\hline - décembre & .6 & $=$ \\
\hline L'image du métier a évolué positivement & 52 & 18 \\
\hline négativement & 2 & 26 \\
\hline $\begin{array}{l}\text { L'image de l'administration Police national } \\
\text { a évolué } \\
\text { positivement } \\
\text { négativement }\end{array}$ & $\begin{array}{l}29 \\
16\end{array}$ & $\begin{array}{r}6 \\
51\end{array}$ \\
\hline $\begin{array}{l}\text { Les policiers sont bien formés, motivés et } \\
\text { l'organisation des services est efficace }\end{array}$ & 35 & 3 \\
\hline
\end{tabular}

En conséquence, l'investissement professionnel des uns et des autres ne sera pas le même : une majoritê des mécontents (56\%) disent en fin de scolarité que s'ils avaient pu ils auraient fait un autre choix, et ils sont $38 \%$ à affirmer que s'ils en ont l'occasion ils quitteront la Police nationale et, bien sûr, ils conseillent moins que les autres d'y entrer.

Tout autant qu'insatisfait, le groupe 6 est déçu par la formation qu'il a reçue, sans doute parce que cette année de scolarité lui a révélé des facettes du métier qui l'attirent peu: par exemple, $38 \%$ des recrues de ce groupe considèrent qu'assurer la sécurité à la sortie des écoles est une tâche indue (ils sont $24 \%$ seulement chez les contents) et $63 \%$ (contre $53 \%$ ) pensent que lors de grands événements sportifs, c'est aux organisateurs de prendre les mesures de sécurité nécessaires. Ils sont $57 \%$ à faire comme choix d'affectation en polices urbaines une brigade de lutte contre la délinquance (contre $49 \%$ dans le groupe opposé) et surtout ils ne sont que $19 \%$ à opter pour le roulement (service général), contre $33 \%$. En fait, ils sont attirés par 
Tableau 3 :

Quelques chiffres relatifs à l'investissement professionnel

\begin{tabular}{lrr}
\hline & Groupe 1 & Groupe 6 \\
\hline S'il avait pu, il aurait fait un autre choix : & & \\
- janvier & 26 & 32 \\
- décembre & 29 & 56 \\
& +3 & +24 \\
\hline Sera policier toute sa vie & 81 & 61 \\
\hline Conseille d'entrer dans la police : & & \\
- janvier & 87 & 87 \\
- décembre & 89 & 64 \\
& $\mathbf{+ 3}$ & $\mathbf{- 2 3}$ \\
\hline
\end{tabular}

les dimensions physiques et répressives du métier : on remarque chez eux une augmentation nettement plus forte de la préférence accordée aux missions de lutte contre la délinquance $(+11$ points contre +2 chez le groupe opposé); le tir et le sport recueillent ensemble $40 \%$ des suffrages relatifs à la matière préférée, contre $26 \%$ chez les satisfaits (et c'est le tir surtout qui fait la différence : $24 \%$ contre $14 \%$ ), alors que les matières juridiques ne rallient que $28 \%$ d'entre eux, contre $44 \%$ chez les contents. Ils sont déçus d'un enseignement dont ils attendaient des recettes et qui leur a donné un bagage juridique dont ils n'ont que faire.

De la même façon, ils sont nettement plus nombreux à penser qu'ils rencontreront souvent des situations de contradiction entre efficacité et respect de la loi.

En parfaite cohérence, cette vision d'une police centrée sur la lutte contre le crime, l'idée que la loi et l'efficacité policière ne font pas souvent bon ménage vont de pair avec un faible attachement à la loi : deux fois plus nombreux que les autres à considérer la loi comme une pure contrainte, moins nombreux à l'inverse à considérer le travail au noir comme une infraction sanctionnable, à penser que la première qualité du policier est l'honnêteté, à trouver que les policiers ont les pouvoirs légaux nécessaires. Et ce rapport distant à la loi s'accompagne d'une propension à la désignation de boucs émissaires : moins nombreux à mettre l'augmentation de la délinquance sur le compte de la détérioration de la situation économique et sociale, ils invoquent plus souvent la tolérance de la justice et l'afflux d'immigrés. Du même mouvement, ils sont $24 \%$ (contre $8 \%$ chez les satisfaits) à considérer que c'est envers cette catégorie de la population qu'il faudra être le plus vigilant. 
Tableau 4 :

Quelques chiffres relatifs à la conception des missions de police et du métier

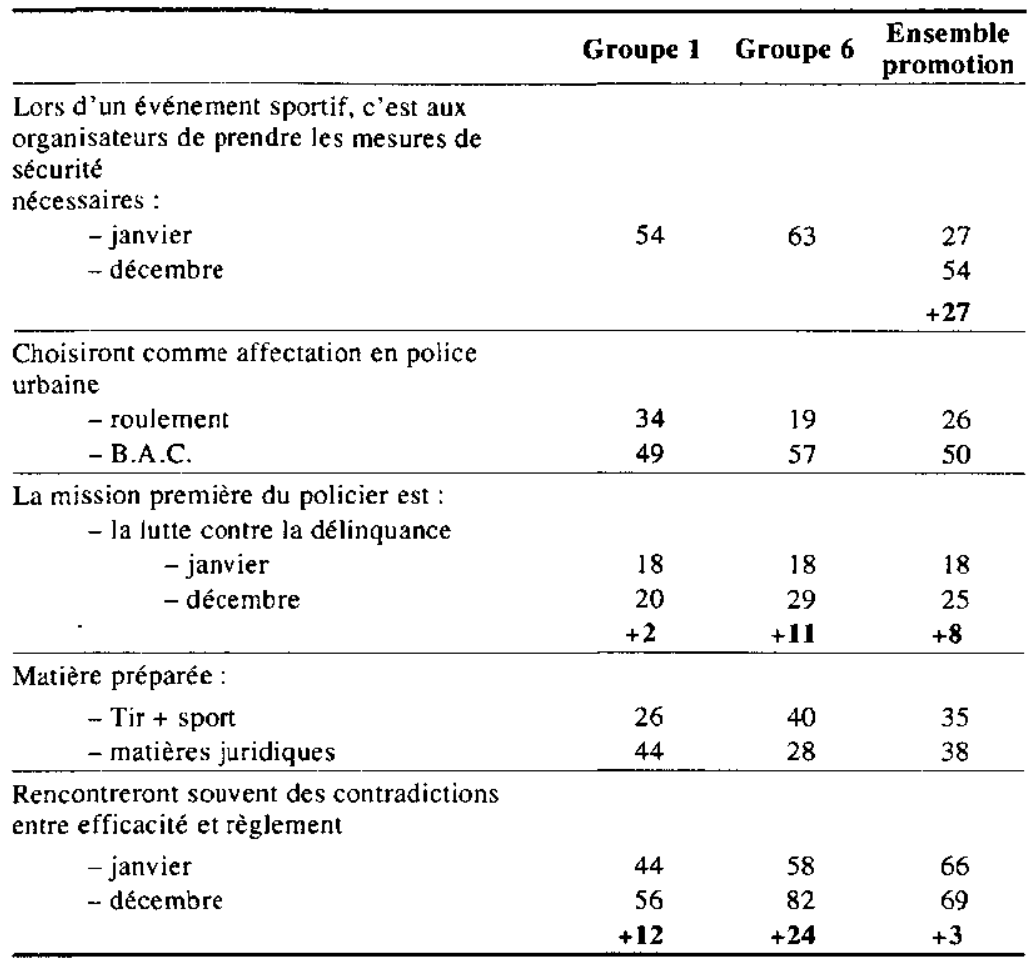

Par contre, sur la deuxième dimension, celle du rapport à l'autre, sur laquelle se positionnent les gardiens (Monjardet, Gorgeon, 1993), contents et mécontents s'opposent peu. Les écarts les plus significatifs (quoique assez faibles) concernent la faute grave du collègue (les mécontents sont $46 \%$ contre $35 \%$ à penser qu'il faut la cacher au public) et la revendication de la prérogative régalienne en matière de police (les contents sont plus tolérants à l'égard des polices municipales: de $74 \%$ en janvier, ils sont encore $61 \%$ à en avoir une opinion positive, tandis que les mécontents ne sont plus que $46 \%$ à avoir cette même opinion (contre $70 \%$ en janvier).

L'opposition quant à la satisfaction vis-à-vis de la formation est donc en réalité à mettre en relation avec l'idée du métier et de la police que se faisaient les uns et les autres et la façon dont cette idée a évolué. 
Tableau 5 :

Quelques chiffres relatifs à la conception et au rapport à la loi

\begin{tabular}{|c|c|c|c|c|}
\hline & \multicolumn{2}{|c|}{ Groupe 1} & \multicolumn{2}{|c|}{ Groupe 6 } \\
\hline \multicolumn{5}{|l|}{ Acception de la loi : } \\
\hline - contrainte & \multicolumn{2}{|c|}{13} & \multicolumn{2}{|c|}{36} \\
\hline - contrat & \multicolumn{2}{|c|}{35} & \multicolumn{2}{|c|}{31} \\
\hline - cadre & \multicolumn{2}{|c|}{27} & \multicolumn{2}{|c|}{15} \\
\hline \multicolumn{5}{|l|}{ Le travail au noir est un délit sanctionnable } \\
\hline- janvier & \multicolumn{2}{|c|}{71} & \multicolumn{2}{|c|}{48} \\
\hline - décembre & \multicolumn{2}{|c|}{70} & \multicolumn{2}{|c|}{48} \\
\hline La première qualité du policier est l'honnêteté & \multicolumn{2}{|c|}{73} & \multicolumn{2}{|c|}{55} \\
\hline Cause de l'augmentation de la délinquance : & janvie & décembre & janvier & décembre \\
\hline - situation économique et sociale & 64 & 64 & 57 & 47 \\
\hline - afflux d’immigrés & 13 & 13 & 14 & 21 \\
\hline - tolérance de la justice & 14 & 7 & 16 & 19 \\
\hline
\end{tabular}

Catégorie envers laquelle il faut être le plus vigilant :

- immigrés

8

24

Ce sont bien là deux modèles de police qui s'opposent avec, d'un côté, une police de service public de proximité, généraliste (de professionnels) aux missions étendues, et, de l'autre, une police plus spécialisée dans la répression du crime, plus resserrée dans ses missions.

Ainsi, à l'encontre de ce qu'affirment les auteurs anglais et américains, les travaux français semblent démontrer que la période de formation à l'école de police ou à l'académie est loin d'agir uniformément sur l'ensemble des recrues au point de pouvoir décrire un processus homogène de socialisation professionnelle qui affecterait l'ensemble des futurs policiers. Bien au contraire, le pluralisme des opinions, attentes et attitudes à l'égard du métier d'une part et le rapport à la loi et à l'autre qu'entretiennent les recrues d'autre part vont servir de filtre à travers lequel serà «passé", en quelque sorte, le message de la formation initiale.

Et il est à parier que l'expérience de terrain, c'est-à-dire l'année de stage en commissariat, sera reçue selon le même procédé et, en même temps qu'elle rapprochera certainement les recrues de leur aînées en activité, elle renforcera les différences sur les questions clés.

Cette apparente divergence est à mettre sur le compte de ces deux facteurs essentiels que sont d'une part les particularités françaises du recrutement et de la formation des policiers, et d'autre part, l'absence d'analyse un peu systématique et de mesure dans les travaux américains qui ont privilégié des démarches inductives ou ethnographiques. 
En France, les gardiens de la paix, comme tous les policiers, sont tous agents de la fonction publique et à ce titre, tout ce qui concerne leur recrutement, le déroulement de la carrière, la notation et le contrôle obéit aux règles qui régissent les emplois publics au même titre que les agents de la Poste, de l'éducation nationale, des Impôts ou des Douanes.

Comme pour tout fonctionnaire, le recrutement initial s'effectue par concours (unique pour l'ensemble du territoire). Les conditions générales d'accès sont donc les mêmes pour tous les candidats, quel que soit le lieu d'affectation futur. De surcroît, l'affectation de la future recrue ne dépend ni de son origine géographique ni de son désir propre, mais est décidée par l'administration en fonction de ses besoins, concentrés bien évidemment dans la région parisienne et les grandes agglomérations. C'est ensuite en fonction du classement de la recrue à la fin de la scolarité que celle-ci "choisira" parmi les postes offerts celui qui a sa préférence. Autant de données que les élèves ont déjà intégrées dès le moment du concours ou très vite dans les premiers jours de formation.

La formation initiale est la même pour tous. Les gardiens ayant réussi le concours sont ensuite répartis dans différentes écoles ou centres de formation situés sur le territoire mais appartiennent tous à la même promotion (l'affectation, du reste, se fera à l'échelle de la promotion et non à celle de l'école). Ils suivent une scolarité relativement longue de 12 mois dont les contenus, les modalités de contrôle et les épreuves de vérification des connaissances sont définis au niveau national. Il est évident que d'une école à l'autre, selon la personnalité du directeur et la composition de l'équipe pédagogique, des variations existent. Celles-ci concerneront l'ambiance, les rapports entre élèves et formateurs, les relations avec l'environnement aussi bien que le contenu, même si la pédagogie par objectifs (au nombre de $80^{23}$ ) qui découpe et décompose considérablement les messages passés pendant la formation a tendance à uniformiser les enseignements. Pendant cette année de formation, les recrues ont peu de contact avec le terrain, hormis à travers les formateurs qui sont tous des policiers et lors de leur stage pratique de deux semaines à peu près à mi-parcours ${ }^{24}$.

D'où, sans doute, une plus grande homogénéité à l'entrée à l'école dans le profil des recrues que dans des pays à l'organisation policière

23. Les élèves sont formés pendant toute leur scolarité par le même formateur qui leur apprend les 80 objectifs. L'enseignement n'est pas spécialisé par discipline, à l'exception des matières techniques telles que le sport, le tir, l'informatique, les langues...

24. Depuis janvier 1994, la formation initiale des gardiens de la paix a été réformée et ceux-ci suivent maintenant une formation dite «en alternance» pendant laquelle ils effectuent plusieurs aller-retour entre l'école et le terrain. 
fortement décentralisée où recrutement (puis formation) s'effectuent à l'échelle de la force ou du département de police selon des critères locaux.

Mais, par ailleurs, quand on discute des différences de résultats entre les recherches anglo-saxonnes et les recherches françaises sur le thème de la socialisation professionnelle, il faut faire la part des méthodes utilisées. Les travaux américains ont fait la part belle à l'observation, voire l'observation participante, et ont privilégié la démarche qualitative qui a indiscutablement permis de faire progresser la compréhension de ces mécanismes complexes de socialisation. Mais en contrepartie, ce que ces démarches ont gagné en compréhension, elles l'ont perdu en représentativité ${ }^{25}$. En effet, le principal défaut de ces approches est qu'elles ne reproduisent que le discours dominant d'une promotion ou d'une école. Par dominant, il faut entendre le discours qui s'exprime avec le plus de force (plutôt que statistiquement majoritaire).

Un minimum de quantification remet en effet ce discours à sa juste place : il est celui d'un quart (grosso modo) des promotions étudiées dans les recherches françaises présentées ici.

\section{BIBLIOGRAPIIIE}

AHERN, F. (1972), Police in Trouble, New York : Hawthom.

BENNETT, R. (1984), «Becomming blue : a longitudinal study of police recruit occupational socialization ", Journal of Police Science and Administration, 12 (1): 47-58.

BUGNON, F. (1994), La socialisation des jeunes gardiens de la paix, Paris.

FIELDING, N. (1988), Joining Forces. Police training, socialization and occupational competence, London : Routledge.

HARRIS, R. (1973), The Police Academy : An lnside View, New York : John Wiley.

HAUSER, G., TIÉVANT, S. (1985), 67451 policiers, Sociographie des personnels de la Police Nationale, Paris, Interface.

LOUBET DEL BAYLE, J. L. (1988), Les élèves gardiens et leur métier, CERP-IEP de Toulouse.

MANNING, P. K. et VAN MAANEN, J. (1978), Policing : a view from the Street, Santa Monica, CA : Goodyear.

MONJARDET, D., GORGEON, C. (1992), 1167 recrues : description de la $121^{\circ}$ promotion des élèves gardiens de la paix de la Police Nationale, Paris : Institut des Haute Études de la Sécurité Intérieure.

25. Comme d'ailleurs la recherche de Françoise Bugnon sur la socialisation des jeunes gardiens de la paix à Paris, fondée pour l'essentiel sur des entretiens avec des gardiens affectés à la préfecture de police, qui apporte des résultats très proches de ceux des auteurs nord-américains (Bugnon, 1994). 
MONJARDET, D., GORGEON, C. (1993), La socialisation professionnelle des policiers - Étude longitudinale de la $12 I^{e}$ promotion des élèves gardiens de la paix. Tome 1, La formation initiale. Paris : Institut des Haute Études de la Sécurité Intérieure.

NIEDERHOFFER, A. (1967), Behind the Shield, New York: Doubleday.

REINER, R. (1985), The Politics of The Police, Harvester Press.

SKOLNIK, J. (1966), Justice without Trial, New York : John Wiley.

STERLING, J. W. (1972), Changes in Role Concepts of Police Officers, Washington D. C. : International Association of Chiefs of Police.

TIFFT, L. (1974), "The "Cop Personnality" reconsidered", Journal of Police Science and administration 2 (3) : 266-78.

VAN MAANEN, J. (1973), "Observations on the Making of policemen», Human Organisations, 32 (4): 407-18.

VAN MAANEN, J. (1975), «Police socialization : a longitudinal examination of job attitudes in a urban police department ", Administration Science Quarterly 20 : 207-28.

WESTLEY, W. A. (1970), Violence and the Police: A sociological Study of Law, Custom and morality, Cambridge, MA/MIT Press. 